\title{
Aspectos do sono e das funções neurocomportamentais em condutores profissionais de veículos pesados: revisão da literatura
}

\author{
Aspects of sleep and neurobehavioral functions in \\ professional drivers of heavy vehicles: literature review
}

\author{
Carlos Souto dos Santos Filho', Gisele Mussi ${ }^{2}$, Eduardo Costa Sá ${ }^{3}$, Vilma Leyton ${ }^{4}$
}

Santos Filho CS, Mussi G, Sá EC, Leyton V. Aspectos do sono e das funções neurocomportamentais em condutores profissionais de veículos pesados: revisão da literatura/ Aspects of sleep and neurobehavioral functions in professional drivers of heavy vehicles: literature review. Rev Med (São Paulo). 2011 abr.-jun.;90(2):78-88.

RESUMO: Caminhoneiros tipicamente têm horários de trabalho irregulares e devem freqüentemente inverter o ciclo vigília-sono, levando a privação parcial de sono cronicamente. Esta situação predispõe a elevados níveis de estresse, ao uso abusivo de drogas e psicoestimulantes, aos distúrbios alimentares, ao sedentarismo e ao desenvolvimento de doenças crônicas. Este estudo teve por objetivo revisar os distúrbios de sono e as alterações neurocomportamentais nesta população. Os autores realizaram uma discussão de 38 artigos selecionados nas bases de dados Medline, Cochrane Library, Lilacs e SciELO, usando como descritores transtornos do sono, condução de veículo, manifestações comportamentais no período de 1997 a 2010. Os autores enfatizaram neste trabalho os aspectos epidemiológicos, os fatores relacionados, os protocolos de avaliação, os instrumentos usados, as modalidades de tratamento, os distúrbios neurocomportamentais associados e as repercussões no trabalho, na saúde e na sociedade. Uma avaliação completa por profissionais capacitados na área de saúde ocupacional é fortemente recomendada na admissão, exames periódicos e na demissão destes funcionários. Faz necessário mais estudo para delinear quais os tipos de protocolos mais úteis na prática clínica, a fim de desenvolver estratégias de tratamento que contemple as necessidades destas pessoas.

DESCRITORES: Transtornos do sono; Manifestações neurocomportamentais; Condução de veículo; Veículos automotores.
ABSTRACT: Truck drivers have typically irregular working hours and they must frequently reverse the sleep-wake cycle which leads them to a chronic partial sleep privation. This situation tpredispose them to high levels of stress, to the use of psycho stimulants and drugs to eating disorders and to sedentarism and the development of chronic diseases. This study aimed to review the sleep-wake cycle and the neurobehavioral changes in this population. The authors performed a discussion of 38 selected articles on Medline, Cochrane Library, Scielo and Lilacs, based on descriptors such as sleep disorders, vehicle driving, behavioral manifestations during the period 1997 to 2010. The authors emphasized on this study epidemiological aspects related factors, the evaluation protocols, instruments used, methods of treatment to neurobehavioral disorders and their impact on labor, health and society. A full analysis to look for qualified professionals in the field of occupational health is strongly recommended for admission, periodic examinations and for a dismissal of those employees. More study is needed to delineate which types of protocols most be useful in clinical practice, to develop treatment strategies that addresses the needs of this population.

KEYWORDS: Sleep disorders; Neurobehavioral manifestations; Automobile driving; Motor vehicles.

\footnotetext{
1. Especialização em Medicina do Trabalho no Departamento de Medicina Legal, Ética Médica e Medicina Social e do Trabalho da Faculdade de Medicina da Universidade de São Paulo (FMUSP) e Médico Fisiatra do IMREA do Hospital das Clínicas da FMUSP.

2. Ergonomista e Assistente Técnica do Serviço de Saúde Ocupacional do Hospital das Clínicas da FMUSP e Doutora em Ciências, Saúde do Trabalhador pela FMUSP.

3. Médico do Trabalho Chefe Il do Serviço de Saúde Ocupacional do Hospital das Clínicas da FMUSP e Oftalmologista. Mestre em Ciências pela Faculdade de Saúde Pública da Universidade de São Paulo.

4. Farmacêutica Bioquímica. Professora Doutora do Departamento de Medicina Legal, Ética Medica e Medicina Social e do Trabalho da FMUSP.

Endereço para correspondência: Carlos Souto dos Santos Filho. Serviço de Saúde Ocupacional (SSO) do Instituto Central do HCFMUSP. Av. Dr. Enéas de Carvalho Aguiar, 155 - 4ํandar, Bloco 21 - Prédio dos Ambulatórios. São Paulo, SP. E-mail: carlos.filho@ hcnet.usp.br
} 


\section{INTRODUÇÃO}

\section{Transtornos do sono e saúde ocupacional}

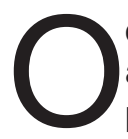
comportamento humano nas atividades rotineiras vem mudando progressivamente com piora persistente na qualidade de vida, no trabalho e no sono. Como o trabalho é uma atividade que pode ocupar grande parcela do tempo de cada indivíduo e do seu convívio em sociedade, condições clínicas do sono podem influenciar quadros de insatisfação até exaustão, aumentar os níveis de absenteísmo, gerando licenças por auxílio-doença e necessidade, por parte da organização, de reformas no quadro de funcionários, despesas extras, entre outras adaptações.

A sociedade contemporânea tem o potencial de promover distúrbios do ritmo circadiano do sono e de comprometimento cognitivo, resultando em eficiência laboral reduzida e insatisfação com possível desenvolvimento de depressão. Por exemplo, sono insuficiente é capaz de alterar o estado de saúde e de promover impactos negativos na economia. A prevalência deste na população geral é estimada em $25 \%$, constituindo-se numa patologia crônica freqüente entre trabalhadores ${ }^{1}$. Outro exemplo são as conseqüências da privação de sono que leva a sonolência diurna excessiva com possível aumento de acidentes de tráfego e nas indústrias, eventos estes com elevado perigo de vida, além de problemas de memória e de atenção'. Compreender a influência do sono insuficiente na função cognitiva e psíquica é fundamental para os profissionais de saúde ocupacional.

Pesquisadores citaram como fatores de risco relevantes para acidentes de trabalho a sonolência e atenção reduzida. Estes autores também relataram dados estatísticos norte-americanos de 1998, onde mais de $52,5 \%$ de todos os acidentes ocorridos no trabalho foram potencialmente associados à sonolência. Desta forma, concluíam que sonolência e outros distúrbios do sono têm forte associação com acidentes de trabalho? ${ }^{2}$

Ao estudar a relação de trabalho por turnos e noturno com as patologias do sono, estudiosos publicaram a influência destes na sociedade moderna, alterando os meios de produção e da organização do trabalho nas indústrias e mudando de forma drástica o estilo de vida, a saúde e o bemestar dos colaboradores ${ }^{3}$. Também mencionaram que turno contínuo e prolongado principalmente a noite predispõe os trabalhadores a doenças cardiovasculares, gastrointestinais e estresse psicossocial. Trabalhadores são rotineiramente submetidos à privação parcial crônica do sono devido ao desacordo recorrente entre o tempo de serviço e os horários fisiológicos de dormir. Sono mais curto e mais fragmentado implica em sonolência excessiva, fadiga mental, irritabilidade e redução do desempenho, que muitas vezes são fatores de risco para acidentes laborais ${ }^{3}$.

Determinadas profissões podem acarretar mais distúrbios do sono. Os condutores profissionais de veículos pesados têm mais chances de apresentar tais distúrbios. Existe grande investimento em pesquisas destes trabalhadores, pois transtornos do sono podem repercutir em grave morbidade e altos índices de acidentes de trânsito.

Ainda existem poucas revisões sobre os aspectos do sono em motoristas comerciais de veículos pesados. O maior conhecimento sobre este assunto nestes trabalhadores será de grande valia para a melhoria do cuidado relativo à prevenção e ao tratamento dos transtornos do sono. Com base nestes dados justifica a realização de uma revisão da literatura sobre o tema.

\section{MATERIAL E MÉTODOS}

Foi realizado estudo tipo revisão de literatura utilizando-se a base de dados Medline, Cochrane Library, Lilacs e SciELO. Utilizaram-se os unitermos: sleep disorders, neurobehavioral manifestations, automobile driving, motor vehicles (e também nas línguas portuguesa e espanhola). A busca foi feita no período de 1997 a 2010. Selecionaram-se artigos empíricos, epidemiológicos, conceituais e de revisão que relacionassem os transtornos do sono e alterações neurocomportamentais em condutores de veículos pesados. Foram excluídos artigos repetidos nas bases de dados, em outras línguas, estudos com crianças e adolescentes e artigos com modelo animal. Numa primeira etapa fez-se levantamento com os quatro descritores acima mencionados. Mas, foram obtidos poucos artigos sobre o assunto. Posteriormente, foi realizada associação com dois descritores na busca de artigos (sleep disorders; automobile driving), obtendo-se 268 artigos e selecionados 38 para a construção deste estudo. A seleção foi baseada na relação com o tema, na qualidade metodológica e na relevância das publicações.

\section{RESULTADOS}

\section{Aspectos gerais da segurança rodoviária mundial}

Atualmente a falta de segurança rodoviária 
é um problema de saúde pública que motivou ações globais de atenção pelas Nações Unidas e pela Organização Mundial da Saúde. As estatísticas mundiais de mortes nas rodovias são preocupantes, por exemplo, em 1998, mais de 1,2 milhões de pessoas em todos os continentes tiveram causa de morte por acidentes de trânsito, sendo a décima maior taxa de mortalidade no mundo ${ }^{4}$. Além disto, muitas pessoas que tiveram acidentes nas estradas adquiriram múltiplas seqüelas. Os custos de assistência médica e outros associados aos acidentes de trânsito são muito elevados devido às graves lesões e suas incapacidades. Estima-se um percentual de 1 a 3\% de investimento do produto interno bruto do país no suporte econômico destes acidentes. Anualmente os Estados Unidos gastam cerca de 518 bilhões de dólares em custos secundários aos acidentes rodoviários ${ }^{4}$.

Estimativas apontam que em 2020, o número de mortos em acidentes de viação deverá praticamente dobrar para 2,3 milhões ${ }^{4}$. Autores referiram um estudo do Reino Unido que mostrou uma prevalência de 1.828 acidentes de trânsito rodoviários em 15 estradas diferentes ${ }^{4}$. Relataram que $17 \%$ dos acidentes graves estavam relacionados à sonolência com uma variação de 3 a $30 \%$, dependendo do tipo de estrada 4 .

Existe uma tendência em estudar a associação da fadiga com acidentes de trabalho em condutores de caminhão, principalmente nos de longa distância. Este interesse cresceu devidos dados alarmantes de mortes no transito nesta classe de trabalhadores. A patrulha rodoviária californiana publicou que o cansaço era responsável por $67 \%$ dos acidentes de caminhão, já o Reino Unido informou que 10 a $25 \%$ das colisões de caminhões foram por fadiga ${ }^{5}$. Pesquisadores citaram uma pesquisa com 107 caminhoneiros americanos, onde a fadiga foi responsável por $58 \%$ das colisões e 19 condutores referiram sonolência no volante. Também mencionaram que na Finlândia, os condutores de caminhões de reboque foram responsáveis por $16 \%$ de todos os acidentes fatais entre 1991 e 1997 e em Israel, as taxas de mortalidade nestes profissionais chegaram a $20 \%$ nos últimos anos e mais de $90 \%$ tiveram colisão com segundo veículo ${ }^{5}$. Portanto, a fadiga é altamente prevalente nestes indivíduos sendo associadas com más condições laborais, longas horas no trafego, doença crônica e transtornos do sono ${ }^{5}$.

No Brasil há predominância maciça do transporte rodoviário, sendo o mesmo responsável pela movimentação de $\mathrm{R} \$ 42$ bilhões ao ano, criando 1,2 milhões de empregos, sendo que mais da metade da população brasileira usa diariamente as estradas para transporte de cargas e passageiros ${ }^{6}$. Em função da opção pela modalidade de transporte rodoviário, da precariedade das vias, da falta de condições físicas dos motoristas e da frota obsoleta, tem-se um quadro assustador de acidentes de trânsito neste país. Dados do Conselho Nacional de Trânsito, em 2001 e 2002, o fator distúrbio do sono foi responsável por 1.787 e 1.844 acidentes, respectivamente. Porém, ao analisar como agente a desatenção, tem-se em 2001, 35.999 acidentes e em 2002, 37.722, o que corresponde, em ambos os períodos, a $35 \%$ do total de acidentes, sendo assim a falta de atenção o maior fator de risco isolado de acidentes neste período ${ }^{6}$.

\section{Aspectos do sono em condutores profissionais de veículos pesados}

Em relação à dinâmica de trabalho imposta aos motoristas comerciais, estudos revelaram as péssimas condições laborais que estes indivíduos enfrentam ${ }^{7,8}$. Caminhoneiros tipicamente têm horários de trabalho irregulares e devem freqüentemente inverter o ciclo vigília-sono, dormindo de dia e permanecendo acordado à noite, levando a privação parcial de sono cronicamente ${ }^{7}$. Esta situação predispõe a elevados níveis de estresse, ao uso de abusivo de drogas e medicamentos psicoestimulantes ${ }^{8}$, aos distúrbios alimentares ${ }^{7}$, ao sedentarismo ${ }^{8}$, ao desenvolvimento de doenças crônicas ${ }^{7}$.

Um estudo americano sugeriu dois fatores que podem estar envolvidos no tema sonolência e acidentes de transito em motoristas profissionais de veículos pesados. O primeiro é sono insuficiente crônico e o outro é a Síndrome da Apnéia Obstrutiva do Sono (SAOS) ${ }^{9}$.

Outra pesquisa constatou que $40 \%$ dos caminhoneiros de longa e $21 \%$ de curta jornada têm dificuldade para se manter alertas em, no mínimo, $20 \%$ do tempo das viagens. Esta população com distúrbios do sono apresentam taxa de acidentes duas vezes maior do que os não portadores ${ }^{6}$.

Diante da hipótese de que a suscetibilidade para acidentes rodoviários associa-se ao incremento de sono não-fisiológico, avaliaram-se a relação da sonolência em motoristas comerciais e disfunção cognitiva ${ }^{10}$, sendo posteriormente confirmada tal hipótese em outro estudo ${ }^{11}$. De fato, pesquisas têm demonstrado uma relação direta entre sonolência excessiva e acidentes com veículos comerciais pesados $^{6,12}$.

Aspectos epidemiológicos dos transtornos do sono

Em relaçãoà epidemiologia das anormalidades do sono em motoristas profissionais, a prevalência 
destes é muito variada na literatura. Isto pode ser um reflexo da falta de critérios de avaliação, poderia também ser o resultado da percepção pessoal enviesada dos profissionais de saúde que atendem esta população.

Nos Estados Unidos, aproximadamente 5.600 pessoas são mortas anualmente em acidentes envolvendo caminhões ${ }^{11}$. Diante desta realidade, pesquisadores do Centro do Sono e Neurobiologia Respiratória da Universidade da Pensilvânia (Filadélfia) realizaram um estudo com objetivo de verificar os fatores de riscos nesta população ${ }^{11}$. Em 406 caminhoneiros, apnéia do sono foi pesquisada de acordo com a gravidade: leve em $17,7 \%$, moderada em $5,8 \%$ e severa em $4,7 \%{ }^{11}$.

SAOS é uma condição clínica causadora de sonolência excessiva e freqüente em caminhoneiros ${ }^{13,14}$. Esta patologia quando não tratada, pode aumentar o risco de acidente rodoviário em 2 a 7 vezes nestes trabalhadores ${ }^{14}$. Com objetivo de validar o consenso americano para triagem de condutores com SAOS, pesquisadores estudaram 1443 motoristas comerciais $^{13}$. Destes 190 foram triados positivos para SAOS e 40 tinham quadro leve, 34 moderado e 53 severo por estudo de polissonografia (PSG) ${ }^{13}$. Outros utilizaram a Escala de Sonolência de Epworth (ESE) para triagem e encontraram $25 \%$ com escore acima de 10 em 843 caminhoneiros americanos ${ }^{15}$. Outro estudo da Universidade da Pensilvânia investigou SAOS em 1329 motoristas comerciais e constataram que 551 tinham alto risco para apnéia do sono por critérios clínicos ${ }^{16}$.

Um estudo caso-controle do Sul da Suécia encontrou $17 \%$ de SAOS em 161 motoristas profissionais. E na analise estatística observaram que os condutores comerciais relataram maior débito do sono que os controles, sendo este resultado estatisticamente significante ${ }^{2}$. Não foram encontrados outros dados de prevalência na Europa nos artigos triados.

Um inquérito australiano verificou após entrevista com 2.342 condutores comerciais e em 161 exames de PSG que 59,6\% tinham distúrbios respiratórios do sono e $15,8 \%$ tinham critérios de SAOS, além de $24 \%$ tinham sonolência excessiva ${ }^{17}$. Já em outra pesquisa com 153 motoristas comerciais israelenses, $77,7 \%$ tinham SAOS, $47,1 \%$ estavam sonolentos, $19 \%$ tinham sonolência severa e $28,1 \%$ sonolência moderada ${ }^{18}$.

Inquérito em 4.331 motoristas profissionais da Tailândia evidenciou sonolência em $75 \%$ e cochilos em $28 \%$ ambos na condução dos veículos ${ }^{19}$. Destes $45 \%$ tinham sonolência excessiva diurna (ESE escore maior que 11) associada fortemente à sensação de sonolência, cochilos e acidentes.
Em 216 condutores de ônibus de Hong Kong foram aplicados questionários específicos para sono e monitoramento do sono por equipamento portátil ${ }^{14}$. Os resultados mostraram que sonolência diurna estava presente em $40 \%$, ronco maior ou igual a 3 vezes por semana em $37 \%$, apnéia testemunhada em $7,9 \%$ e $13,4 \%$ referiram ter adormecido durante a condução ${ }^{14}$. Entre os 51 indivíduos que realizaram avaliação domiciliar do sono através de equipamento portátil, $61 \%$ tiveram Índice de Distúrbio Respiratório (IDR) maior ou igual a 5 por hora de sono, $41 \%$ com IDR maior ou igual a 10 por hora de sono, $24 \%$ IDR maior ou igual a 15 por hora de sono e 35 roncava objetivamente maior ou igual a $10 \%$ da noite. Como a definição de distúrbios respiratórios do sono é dada com IDR maior ou igual a 5 , a prevalência foi de $14,4 \%$, enquanto que a freqüência de SAOS (definida como IDR maior ou igual a 5 mais sonolência relatada) foi de $4,6 \%{ }^{14}$.

Outro estudo em Hong Kong com 1.016 motoristas de ônibus verificou sonolência no trabalho em $60,9 \%$, sendo que $23,9 \%$ tinham ronco maior ou igual a três noites por semana, 3,7\% de apnéia testemunhada e $24 \%$ de adormecimento durante a condução ${ }^{20}$. Entre os 211 participantes que se submeteram ao estudo do sono em casa, $40,3 \%$, $26,1 \%$ e $17,5 \%$ tinham IDR maior ou igual a 5 , maior ou igual a 10 e maior ou igual a 15 por hora, respectivamente ${ }^{20}$.

Estudo transversal comparativo realizado em Lima (Peru), de um total de 271 motoristas informais e 71 formais, uma porcentagem de $16 \%$ tinha sonolência ${ }^{21}$. Na argentina, 738 caminhoneiros foram entrevistados, destes $43,7 \%$ tinham sonolência na condução com ESE maior que $10^{22}$. Já ronco foi prevalente em $71 \%$, sendo freqüente em $43,8 \%$. Ronco mais de 3 vezes por semana, sonolência ao dirigir e ESE maior que 10 foram independentemente associados com acidentes ou quase acidentes ${ }^{22}$.

No Brasil, a literatura encontrada nos bancos de dados utilizados é vasta em relação aos distúrbios do sono e sua prevalência nesta população. Uma proporção relevante das pesquisas avaliou motoristas de veículos pesados, obtendo dados muitas vezes semelhantes.

Em 4.878 caminhoneiros do Estado de São Paulo, 70,4\% destes com índice de massa corporal (IMC) menor que 30 e outros $29,6 \%$ com IMC maior que 30 tinham sono curto 7 . Em $63,4 \%$ com IMC menor que 30 e $36,6 \%$ com IMC maior que 30 tinham ronco. E em $38,2 \%$ com IMC menor que 30 e $61,8 \%$ com IMC maior que 30 tinham alto risco para SAOS ${ }^{7}$. Viegas e Oliveira ${ }^{6}$ encontraram em 262 condutores de ônibus em linhas interestaduais brasileiras $27,5 \%$ de hipersonolentos e $35 \%$ de hipersonolentos com 
IMC maior que $30 \mathrm{~kg} / \mathrm{m}^{2}$. Pesquisadores ${ }^{12}$ aplicaram o Índice de Qualidade do Sono de Pittsburgh (IQSP) em 260 caminhoneiros brasileiros. Este questionário é capaz de avaliar subjetivamente a qualidade do sono e identificar os distúrbios do sono nos últimos 30 dias. Observaram que a média do IQSP foi de $4,95( \pm 2,56)$. Em $35,4 \%$ tinham o IQSP maior que 5 e $23(11,1 \%)$ sujeitos ressonavam mais que 3 vezes por semana. A pontuação do IQSP foi maior entre aqueles que dormiam menos horas (menos que $5 \mathrm{~h}$ ou 5 a 6 horas) do que no grupo dos motoristas que dormia 7 a 8 horas. Também foi significativamente maior no grupo que acordava mais cedo (antes das 5 da manhã). Em relação drogas, encontraram 50,9\% relatos de uso álcool e $11,1 \%$ de anfetaminas.

Caminhoneiros brasileiros (total de 438) tinham insônia $(26,6 \%)$, ronco alto $(45 \%)$ e apnéia testemunhada $(7,6 \%)^{23}$. E em 392 motoristas, $22 \%$ referiram adormecer durante a condução e $2,8 \%$ relataram adormecer diariamente ou quase diariamente. Em outra população de 209 motoristas brasileiros de caminhão a prevalência de SAOS foi de $11,5 \% ; 34,5 \%$ referiram dormir ao volante enquanto dirigiam ao menos uma vez e $38,7 \%$ referiram roncar durante o sono ${ }^{24}$.

Em 32 motoristas de ônibus brasileiros, 38\% tinham índices de apnéia e hipopnéia (acima de 5 de sono por hora) durante sono noturno e diurno ${ }^{25}$, já ronco diurno foi significativo em $50 \%$ e noturno em $35 \%$. Além disto, 38\% tinham movimentos periódicos dos membros inferiores. Pelo teste de gravação de latências múltiplas do sono, $42 \%$ (dia) e $38 \%$ (noite) tinham critérios para sonolência. Durante dia o sono foi mais curto e mais fragmentado com maior sonolência excessiva. Distúrbio respiratório foi um achado comum em trabalhadores por turnos ${ }^{25}$. Um grande inquérito com 10.101 motoristas brasileiros de caminhão encontrou $26 \%$ de alto risco para SAOS $^{26}$.

\section{Fatores relacionados ao desenvolvimento dos} transtornos do sono

Para a enumeração dos fatores associados ao desenvolvimento dos distúrbios do sono em motoristas comerciais de veículos pesados são levadas em consideração três dimensões: o trabalho, o indivíduo e a sociedade.

\section{Trabalho}

Em relação aos fatores relacionados ao trabalho que influenciam o desenvolvimento do sono não fisiológico nesta população, alguns itens são mencionados na Tabela 1.

\section{Indivíduo}

Acredita-se que características próprias do indivíduo podem estar associadas a maiores índices de transtornos do sono em motoristas comerciais de veículos pesados (Tabela 2).

\section{Sociedade}

Os fatores sociais associados aos problemas de sono em profissionais condutores são apresentados na Tabela 3.

TABELA 1. Fatores laborais (características do trabalho) associados aos transtornos do sono

\begin{tabular}{|c|c|}
\hline Fator & Possíveis conseqüências \\
\hline $\begin{array}{l}\text { Cochilo após jornada noturna } \\
\text { prolongada }\end{array}$ & $\begin{array}{l}\text { Oportunidade de cochilar durante } 3 \text { horas no período vespertino diminuiu a } \\
\text { sonolência noturna e a fadiga em motoristas de longas distâncias }{ }^{27} \text {. }\end{array}$ \\
\hline Sonolência (ESE >10) & Aumenta risco de acidentes de trânsito 22,23 . \\
\hline Sobrecarga e jornada de trabalho & $\begin{array}{l}\text { Jornada acima de } 12 \text { horas e sem dias de folga em } 61 \% \text { de motoristas comerciais } \\
\text { estava relacionada à alteração sono e risco maior de acidentes }{ }^{19} \text {. Estudo } \\
\text { argentino }^{22} \text { e israelense } \text { também fizeram estas associações. }^{5}\end{array}$ \\
\hline Trabalho por turnos e noturno & $\begin{array}{l}\text { Relação com sonolência em motoristas comerciais e indiretamente risco de } \\
\text { acidente }^{19,25} \text {. }\end{array}$ \\
\hline Tipo de vínculo empregatício & $\begin{array}{l}\text { SAOS em caminheiros brasileiros estava fortemente associada ao vínculo } \\
\text { informal, IMC maior ou igual a } 25 \mathrm{~kg} / \mathrm{m}^{2} \text { e qualidade do sono ruim, interferindo } \\
\text { na saúde destes trabalhadores }{ }^{24} \text {. }\end{array}$ \\
\hline
\end{tabular}


Santos Filho et al. Aspectos do sono e das funções neurocomportamentais.

TABELA 2. Fatores individuais associados a índices superiores de transtornos do sono

\begin{tabular}{|c|c|}
\hline Fator & Características \\
\hline Obesidade & $\begin{array}{l}\text { Motoristas comerciais obesos têm menor duração sono. Ambos estão } \\
\text { associados independentemente a problemas de saúde (distúrbios } \\
\text { endócrinos, Hipertensão Arterial Sistêmica e ronco) }{ }^{7} \text {. Motoristas obesos } \\
\text { tinham maiores índices de sonolência excessiva diurna }{ }^{6,19} \text {, apnéia do } \\
\text { sono }^{16} \text { e SAOS } \\
1,18,20,24\end{array}$ \\
\hline Uso abusivo de álcool e drogas & $\begin{array}{l}\text { Uso abusivo destas substâncias pode acarretar problemas psiquiátricos, } \\
\text { distúrbios do sono, dentre outros } \\
\text { álcool e drogas são vulneráveis a menor desempenho na condução de } \\
\text { Tabaco e outras drogas foram associados a alto risco para SAOS }{ }^{26} \text {. }\end{array}$ \\
\hline Uso de medicações para doenças crônicas & $\begin{array}{l}\text { Algumas medicações para doenças crônicas têm efeitos adversos na } \\
\text { arquitetura do sono }{ }^{19} \text { e aumentam risco de acidentes no trânsito }{ }^{17} \text {. }\end{array}$ \\
\hline Associação com hábitos ruins & $\begin{array}{l}\text { Ingerir bebidas cafeinadas e energéticos, mascar goma ou lanches } \\
\text { em excesso foram estratégias adotadas para diminuir sonolência na } \\
\text { condução e podem agravar os distúrbios do sono }{ }^{19} \text {. }\end{array}$ \\
\hline Idade & 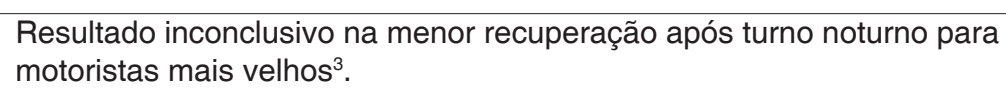 \\
\hline
\end{tabular}

TABELA 3. Fatores sociais associados aos transtornos do sono

\begin{tabular}{l|l}
\hline Fator & \multicolumn{1}{c}{ Características } \\
\hline $\begin{array}{l}\text { Péssimas condições das estradas e dos } \\
\text { locais de repouso }\end{array}$ & $\begin{array}{l}\text { Envolvimento em acidente com vítimas foi associada significativamente } \\
\text { com dificuldade freqüente de encontrar locais para descanso }\end{array}$ \\
\hline $\begin{array}{l}\text { Falta de programas educacionais para } \\
\text { incentivar atividade física }\end{array}$ & $\begin{array}{l}\text { Maiores níveis de sedentarismo em caminhoneiros com SAOS e pouco } \\
\text { investimento em educação em saúde }\end{array}$ \\
\hline Forma de contrato trabalho & $\begin{array}{l}\text { Contratos informais são freqüentes em motoristas de caminhão e } \\
\text { foram associados à SAOS }{ }^{24} \text {. Já um estudo peruano não mostrou esta } \\
\text { associação entre motoristas formais e informais }{ }^{21} .\end{array}$ \\
\hline Falta de suporte social e familiar & $\begin{array}{l}\text { O ritmo de trabalho e os fatores organizacionais impedem o motorista } \\
\text { profissional de ter maior apoio de amigos e da família, podendo influenciar } \\
\text { o uso abusivo de substâncias }{ }^{8} .\end{array}$ \\
\hline
\end{tabular}

\section{Aspectos clínicos dos transtornos do sono}

Para melhor abordar este assunto, optou-se por dividir em três subtemas.

\section{Protocolos clínico-epidemiológicos}

Examinou-se a inter-relação entre IMC, a gravidade da SAOS e o grau de sonolência excessiva diurna ${ }^{18}$. Constatou-se forte correlação entre obesidade e grau de sono durante o dia. Os relatos subjetivos de SAOS ou dos sintomas de fadiga não são confiáveis. O IMC pode ser um critério de seleção de primeira linha para detectar sonolência excessiva diurna em motoristas profissionais ${ }^{18}$.

Um estudo avaliou prospectivamente cinco estratégias diferentes para selecionar motoristas com apnéia grave em triagem ocupacional ${ }^{16}$. Utilizou-se PSG como teste padrão-ouro para comparação. As formas de avaliação foram apenas sintomas, IMC isoladamente, sintomas mais IMC, dois estágios (sintomas mais IMC seguido de oxímetro) e somente oxímetro. A estratégia dois estágios teve $91 \%$ de sensibilidade e especificidade para identificar apnéia grave em condutores profissionais, podendo ser utilizada em exames admissionais e periódicos.

Três Instituições Americanas (Colégio Americano de Pneumologia, Colégio Americano de Medicina e Meio Ambiente do Trabalho (CAMMAT) e Fundação Nacional do Sono) reuniram-se para criar uma força-tarefa com objetivo de criar um consenso para abordar SAOS em motoristas comerciais ${ }^{28,29}$. Estudaram-se a validação deste consenso em 1443 condutores profissionais ${ }^{13}$. Estes autores encontram $13 \%$ positivos para SAOS. Os resultados sugerem que os critérios adotados pelo consenso são específicos, mas não sensíveis para SAOS ${ }^{13}$.

Com o objetivo de verificar a aceitação deste 
consenso, o CAMMAT aplicou eletronicamente um instrumento breve para seus sócios médicos ${ }^{30}$. Cerca de $92 \%$ dos 552 examinadores opinaram positivamente sobre a importância deste protocolo. Mas, apenas $42 \%$ relataram ter utilizado. As razões apontadas foram: consenso ser inconsistente (36\%), complicado (12\%), demorado $(10 \%)$ e inconveniente para motorista (10\%). Outros autores chamaram a atenção para a ausência de testes neurocomportamentais neste consenso ${ }^{29}$.

\section{Instrumentos de avaliação}

Em Neuropsicologia, o emprego de escalas de avaliação é muito freqüente, o mesmo se dá na área da Medicina do Sono. Nesta revisão encontraram-se diversos questionários, alguns específicos para sono, outros para as funções corticais superiores (cognição) e ainda teste para alterações de comportamento (Escala Tática do Conflito) ${ }^{8}$. Uma escala foi observada para avaliar uso abusivo e dependência álcool $(\mathrm{CAGE})^{8}$, sendo fundamentais para inquéritos clínico-epidemiológicos em condutores comerciais. Questionários sócio-demográficos foram usados em algumas pesquisas $7,14,15,24,28$.

As escalas para funções cognitivas encontradas foram Tarefa Vigilância Psicomotora ${ }^{11,29}$, Tarefa de Dirigir de Atenção Dividida ${ }^{11}$, Teste de Atenção Concentrada ${ }^{6}$, Teste de Atenção Difusa ${ }^{6}$. Um instrumento que classifica os indivíduos quanto freqüência e duração da atividade física e nível de sedentarismo foi aplicado em caminhoneiros brasileiros (International Physical Activity Questionnaire - versão curta $)^{24}$. As escalas relacionadas especificamente ao estudo do sono estão listadas na Tabela 4 com sua aplicabilidade.

TABELA 4. Instrumentos de avaliação utilizados nos trabalhos

\begin{tabular}{l|l}
\hline Instrumentos de avaliação & \multicolumn{1}{c}{ Aplicabilidade } \\
\hline Questionário de Berlim & Discrimina duas categorias: alto e baixo risco para SAOS. \\
\hline Questionário de Previsão de Apnéia Multivariável & Verifica maior risco para a apnéia do sono ${ }^{11}$. \\
\hline QSE & Avalia subjetivamente sonolência ${ }^{31,32}$. \\
\hline Questionário da Matutinidade e Vespertinidade & Avalia alto e moderado risco para apnéia do sono ${ }^{27}$. \\
\hline IQSP & Estabelece o tipo de ritmo circadiano do individuo ${ }^{27}$. \\
\hline Escala de Sonolência Stanford & $\begin{array}{l}\text { Avalia subjetivamente a qualidade do sono e identificar os } \\
\text { distúrbios do sono nos últimos 30 dias }{ }^{12} .\end{array}$ \\
\hline Questionário Saúde e Sono & $\begin{array}{l}\text { De forma subjetiva verifica através do auto-relato sete diferentes } \\
\text { estados de sonolência }{ }^{23} \text {. }\end{array}$ \\
\hline Wisconsin Sleep Cohort Study questionnaire & $\begin{array}{l}\text { Utilizado para caracterizar sintomas de apnéia do sono em } \\
\text { inquéritos populacionais }{ }^{20} .\end{array}$ \\
\hline The Functional Outcomes of Sleep Questionnaire & $\begin{array}{l}\text { Estuda de forma global sono (hábito sono, ronco, movimentos } \\
\text { involuntários, estratégias dos motoristas durante sonolência, } \\
\text { dentre outros) }\end{array}$ \\
\hline
\end{tabular}

\section{Modalidades de intervenção}

Nos artigos revisados existem alguns tratamentos descritos para abordar as patologias do sono em motoristas comerciais de veículos pesados. Os trabalhos variam de uso isolado de mudança de hábitos a abordagens com uso de dispositivos nasais específicos em Centros de Medicina do Sono.

Pesquisados americanos observaram respostas positivas na fadiga e sonolência subjetiva, nos tempos de reação e na variabilidade do desempenho nas tarefas psicomotoras após emprego de 3 horas de cochilo vespertino em situação simulada de condução noturna em oito motoristas profissionais de longa jornada ${ }^{27}$. Utilizaram neste protocolo registros eletrofisiológicos com polissonografia contínua e emprego de questionário para qualidade do sono, sonolência e fadiga. Um estudo coreano investigou o emprego de Pressão Positiva Contínua Nasal domiciliar em motorista de ônibus com apnéia do sono ${ }^{20}$. A aceitação deste dispositivo foi baixa, mas houve melhora significativa da sonolência subjetiva e na função cognitiva entre aqueles que realizaram o tratamento.

Ensaio duplo cego, randomizado e cruzado envolvendo 12 jovens saudáveis teve objetivo de 
testar os efeitos da cafeína (15 miligramas) ou de cochilo (30 minutos) em condução noturna num carro. Os autores concluíram que ingerir café ou cochilar por 30 minutos reduziu de forma significante as desvantagens de direção noturna sem comprometer a qualidade do sono subseqüente ${ }^{33}$.

A vigilância reduzida é comum em condução noturna. Para diminuir a sonolência pode-se empregar exposição de luz. Seis caminhoneiros participaram de experimento com auto-aplicação de luz para testar esta hipótese ${ }^{34}$. Nesta pesquisa tinham que incidir luz por 30 minutos em 10 momentos. Esta intervenção com luz não mostrou efeitos significativos, pois não aumentou a vigilância na situação de direção noturna.

Embora haja um crescente apoio na literatura para garantir que o sono e as questões relacionadas sejam vistas como um elemento central de qualquer protocolo de saúde ocupacional para profissionais do trânsito há ainda pouco investimento para abordar esta problemática dentro dos Programas de Controle Médico de Saúde Ocupacional.

\section{Associação com transtornos neurocomportamentais}

Avanços na Medicina do Sono identificaram repercussões específicas neurocomportamentais que cronicamente pioram a qualidade de vida dos condutores profissionais de veículos pesados. Em virtude da freqüência com que ocorre a SAOS nesta população, as pesquisas focam cada vez mais neste assunto.

Estudo com motoristas comerciais realizou testes cognitivos (lapsos de desempenho e controle de erro em tarefa de dirigir com Atenção dividida) e mostrou que o sono curto de duração crônico é fator de risco para comprometimento do desempenho de conduzir veiculo ${ }^{11}$. Já em 262 motoristas profissionais brasileiros de ônibus interestadual, os que tiveram maiores índices na ESE apresentaram nível de atenção concentrada comprometido, além de relação direta entre a circunferência do pescoço e a hipersonolência associada também a menor nível de atenção difusa ${ }^{6}$.

Estudo experimental francês testou as possíveis conseqüências neurocomportamentais da restrição do sono combinado com a fadiga da condução de longa distância em motoristas comuns em 3 situações diferentes ${ }^{10}$. Os resultados mostraram associação do sono restrito e fadiga com prejuízo significativo no tempo de reação.

Outra pesquisa experimental examinou os efeitos do sono insuficiente sobre a função cognitiva, desempenho na condução e fluxo sangüíneo cerebral em 19 adultos saudáveis ${ }^{1}$. Concluíram que uma noite de sono insuficiente afeta a função cognitiva diurna e a performance de condução, associada a alterações na resposta da oxigenação cortical. E outros verificaram efeitos deletérios nos potenciais evocados visuais, levando ao chamado efeito visão de túnel ${ }^{35}$.

Num estudo duplo-cego e randomizado com 28 pessoas saudáveis, avaliou-se os efeitos residuais de três hipnóticos (15 miligramas de gaboxadol, 7,5 miligramas de zopiclone e 10 miligramas de zolpidem) na função cognitiva, psicomotora e desempenho de direção veicular ${ }^{36}$. Verificaram que o horário de administração destas medicações pode influenciar nas funções corticais.

Outros distúrbios neurocomportamentais nesta população estão relacionados ao uso abusivo ou dependência de substâncias psicoativas, que indiretamente tem associação com os transtornos do sono ${ }^{37}$. Estudos mostraram alta prevalência de uso de álcool e outras drogas ${ }^{12,19,38}$, além da forte participação de fatores psicossociais nesta condição ${ }^{8}$.

\section{Conseqüências dos transtornos do sono}

Muitos pontos permanecem não esclarecidos, mas os autores, de forma geral, concordam que os distúrbios do sono interferem diretamente ou não nos níveis institucional, pessoal e social.

Para a organização, pode acarretar em aumento dos gastos (tempo, dinheiro) com a conseqüente rotatividade de funcionários acometidos pelos transtornos do sono, assim como aumentar nível de absenteísmo. Nenhum estudo investigou estas conseqüências. Outro aspecto importante e muito abordado nas pesquisas foi o risco potencial de acidente de trânsito ${ }^{6,12,23}$. Isto pode acarretar sérios problemas financeiros e jurídicos para os empregadores (organizações).

Para o indivíduo, as conseqüências podem ser decorrentes ou não dos distúrbios do sono. Além disto, estas conseqüências podem retroalimentar negativamente a gravidade de tais distúrbios. Incluem transtornos cardiovasculares ${ }^{7,26}$, respiratórios ${ }^{14,20}$, neuropsiquiátricos ${ }^{6,10,11}$ e neuroendócrinos ${ }^{7,18,19}$. Outros distúrbios não foram encontrados nos artigos.

Em relação às conseqüências na sociedade, esta população enfrenta várias dificuldades psicossociais que agravam os transtornos do sono. Nesta classe de trabalhadores é comum o distanciamento dos amigos e familiares devido às longas jornadas de trabalho ${ }^{8}$. Além disto, o estresse é muito freqüente nesta população ${ }^{8}$. Está associado à falta de segurança nas estradas, aos problemas financeiros e as pressões abusivas dos 
empregadores. O principal impacto na sociedade está relacionado ao alto índice de acidentes de trânsito em motoristas acometidos por patologias do sono, que podem aumentar de forma significativa os gastos com as indenizações, com a assistência de reabilitação física, dentre outros.

\section{DISCUSSÃO}

Nesta revisão sobre aspectos do sono e cognitivo-comportamentais em condutores profissionais de veículos pesados, os fatores relacionados, os dados epidemiológicos e os instrumentos de avaliação foram os temas mais abordados nos artigos. O desenho em revisão de literatura possibilitou verificar o estado da arte do sono nesta população nos temas acima descritos.

Em relação à epidemiologia, observouse uma variabilidade nos dados estatísticos de prevalência nas morbidades do sono em motoristas comerciais. SAOS $2,7,13,16,26$ foi o transtorno mais referido nas pesquisas seguido por distúrbio respiratório do sono ${ }^{14,17}$. Dentre os sintomas relacionados a estes transtornos, sonolência excessiva diurna e fadiga foram os mais prevalentes ${ }^{15,18,19,21}$ e estatisticamente associados a acidentes, sendo um forte fator de risco no trabalho nas estradas ${ }^{22}$.

Alguns autores relatam como possíveis fatores de risco relacionados ao trabalho para desenvolvimento de disfunções do sono a sobrecarga com longas jornadas no trânsito ${ }^{5,19,22}$, trabalho noturno e por turnos ${ }^{19,25}$ e o tipo de vinculo empregatício ${ }^{24}$. Todos estes fatores podem favorecer ao surgimento do transtorno de Burnout, que não foi relatado nos estudos. Já os fatores individuais mais relevantes apontados foram obesidade e uso abusivo de substâncias psicoativas. Estes fatores também estão relacionados a outras comorbidades físicas e psíquicas agravando a qualidade de vida destes trabalhadores. A falta de suporte social e familiar, peculiar a esta profissão, retroalimenta negativamente os transtornos do sono ${ }^{8}$.

O uso da PSG acarretou enorme avanço nas pesquisas de sono, possibilitando melhor avaliação por parâmetros objetivos da arquitetura e função do sono. Foi usada como exame padrão-ouro nas comparações de outras estratégias de avaliação nesta população. Outra estratégia com boa acurácia estudada foi o conjunto formado por sintomas e IMC seguido de oxímetro ${ }^{16}$.

Dentre os instrumentos de pesquisa, 0 mais encontrado nos artigos foi a ESE. Não existe uniformidade na literatura quanto à validade clinica e epidemiológica desta escala. Alguns autores criticam seus resultados por ser uma ferramenta subjetiva e outros acreditam ser viável em triagem ocupacional. Nesta revisão foi encontrado o consenso americano ${ }^{28}$ que já foi validado em estudo posterior com alto valor preditivo positivo para pesquisa de SAOS em motoristas comerciais ${ }^{13}$. Outros autores criticaram este consenso em relação aos critérios utilizados para verificar adesão ao tratamento da SAOS e mencionam que o Dispositivo de Pressão Positiva Contínua nas Vias Aéreas (CPAP) poderia ser mais objetivo e confiável neste objetivo ${ }^{29}$.

Em relação às modalidades terapêuticas, foram encontrados ensaios experimentais com pessoas saudáveis, portadoras de distúrbios do sono, em situações simuladas ou reais. Mas, devido o número pequeno de participantes e a metodologia cientifica empregada não tem evidencia para serem adotados na pratica clínica. Estudo coreano comprovou que CPAP melhorou a sonolência excessiva e a função cognitiva em motoristas de ônibus ${ }^{20}$.

Quanto à associação com transtornos neurocomportamentais, eles estão envolvidos na gênese, na clínica e na indicação do tratamento do sono patológico. Os mais freqüentes são déficits de atenção e desempenho na direção de veículos. Um estudo verificou a diminuição na oxigenação do córtex cerebral em situação de sono insuficiente que justificaria as alterações cognitivas e comportamentais ${ }^{1}$.

Poucas pesquisas clínicas sobre tratamento farmacológico para os transtornos do sono foram encontradas para esta população. Ensaio clínico randomizado controlado por placebo com pessoas saudáveis avaliou os efeitos residuais na capacidade de direção veicular de três hipnóticos não-benzodiazepínicos (gaboxadol, zopiclone e zolpidem ${ }^{36}$. As estratégias de tratamento que podem trazer mais melhorias na função do sono em motoristas comerciais são as intervenções multiprofissionais em Centros Especializados, mas este tipo de intervenção não foi verificado nos artigos.

A mortalidade e morbidade elevadas estão relacionadas à grande prevalência de transtornos do sono em motoristas comerciais. Este forte risco de acidente de transito ocasiona danos a saúde, a instituição e a sociedade. Os transtornos cardiovasculares, respiratórios, neuropsiquiátricos e neuroendócrinos foram os mais referidos pelos autores. Outras patologias poderiam ter sido investigadas tais como dor crônica, depressão, transtornos fóbicos e doenças sexualmente transmissíveis.

As conseqüências sociais dos transtornos do sono na vida destes trabalhadores são pouco contempladas pela literatura. A falta de suporte 
social e familiar, a falta de segurança nas estradas, a pressão abusiva dos empregadores e os problemas financeiros são os problemas mais comuns relatados em pesquisa qualitativa ${ }^{8}$.

\section{CONCLUSÃO}

Os distúrbios do sono são muito prevalentes em motoristas profissionais de veículos pesados. SAOS e distúrbios respiratórios do sono foram os mais freqüentes. Os fatores de risco relacionados a estes transtornos são distribuídos em organizacional,

\section{REFERÊNCIAS}

1. Miyata S, Noda A, Ozaki N, Hara $Y$, Minoshima M, Iwamoto K, Takahashi M, lidaka T, Koike Y. Insufficient sleep impairs driving performance and cognitive function. Neurosci Lett. 2010;469(2):229-33.

2. Carter N, Ulfberg J, Nyström B, Edling C. Sleep debt, sleepiness and accidents among males in the general population and male professional drivers. Accid Anal Prev. 2003;35(4):613-7.

3. Pires ML, Teixeira CW, Esteves AM, Bittencourt LR, Silva RS, Santos RF, et al. Sleep, ageing and night work. Braz J Med Biol Res. 2009;42(9):839-43.

4. Pandi-Perumal SR, Verster JC, Kayumov L, Lowe AD, Santana MG, Pires MLN, et al. Sleep disorders, sleepiness and traffic safety: a public health menace. Braz J Med Biol Res. 2006;39(7):863-71.

5. Sabbagh-Ehrlich S, Friedman L, Richter ED. Working conditions and fatigue in professional truck drivers at Israel ports. Inj Prev. 2005;11(2):110-4.

6. Viegas CAA, Oliveira HW. Prevalência de fatores de risco para a síndrome da apnéia obstrutiva do sono em motoristas de ônibus interestadual. J Bras Pneumol. 2006;32(2):144-9.

7. Moreno CR, Louzada FM, Teixeira LR, Borges F, Lorenzi-Filho G. Short sleep is associated with obesity among truck drivers. Chronobiol Int. 2006;23(6):1295303.

8. Anderson DG, Riley P. Determining standards of care for substance abuse and alcohol use in long-haul truck drivers. Nurs Clin North Am. 2008;43(3):357-65.

9. Pack Al, Maislin G, Staley B, Pack FM, Rogers WC, George CF, et al. Impaired performance in commercial drivers: role of sleep apnea and short sleep duration. Am J Respir Crit Care Med. 2006;174(4):446-54.

10. Philip P, Sagaspe P, Taillard J, Moore N, Guilleminault $\mathrm{C}$, Sanchez-Ortuno $\mathrm{M}$, et al. Fatigue, sleep restriction, and performance in automobile drivers: a controlled study in a natural environment. Sleep. 2003;26(3):27780.

11. Pack Al, Maislin G, Staley B, Pack FM, Rogers WC, George CF, et al. Impaired performance in commercial drivers: role of sleep apnea and short sleep duration. individual e social. Estão mutuamente envolvidos e agravam sobremaneira o prognóstico.

Os protocolos de avaliação são baseados em parâmetros objetivos e subjetivos. Na literatura existe uma predileção pelos objetivos. Não há um consenso brasileiro para avaliar SAOS em motoristas comerciais. A abordagem dos aspectos neurocomportamentais na avaliação dos transtornos do sono é fundamental para uma conduta mais adequada devido sua forte associação. As conseqüências destes transtornos são em nível institucional, individual e social. Estão mutuamente onipresentes na vida destes trabalhadores e condicionam má qualidade de vida.

Am J Respir Crit Care Med. 2006;174(4):446-54.

12. Souza JC, Paiva T, Reimão R. Sleep habits, sleepiness and accidents among truck drivers. Arq Neuropsiquiatr. 2005;63(4):925-30.

13. Talmage JB, Hudson TB, Hegmann KT, Thiese MS. Consensus criteria for screening commercial drivers for obstructive sleep apnea: evidence of efficacy. J Occup Environ Med. 2008;50(3):324-9.

14. Hui DS, Chan JK, Ko FW, Choy DK, Li TS, Chan AT, et al. Prevalence of snoring and sleep-disordered breathing in a group of commercial bus drivers in Hong Kong. Intern Med J. 2002;32(4):149-57.

15. Heaton K, Browning S, Anderson D. Identifying variables that predict falling asleep at the wheel among long-haul truck drivers. AAOHN J. 2008;56(9):37985.

16. Gurubhagavatula I, Maislin G, Nkwuo JE, Pack AI. Occupational screening for obstructive sleep apnea in commercial drivers. Am J Respir Crit Care Med. 2004;170(4):371-6.

17. Howard ME, Desai AV, Grunstein RR, Hukins C, Armstrong JG, Joffe D, et al. Sleepiness, sleepdisordered breathing, and accident risk factors in commercial vehicle drivers. Am J Respir Crit Care Med. 2004;170(9):1014-21.

18. Dagan Y, Doljansky JT, Green A, Weiner A. Body Mass Index (BMI) as a first-line screening criterion for detection of excessive daytime sleepiness among professional drivers. Traffic Inj Prev. 2006;7(1):44-8.

19. Leechawengwongs $M$, Leechawengwongs $E$, Sukying $\mathrm{C}$, Udomsubpayakul U. Role of drowsy driving in traffic accidents: a questionnaire survey of Thai commercial bus/truck drivers. J Med Assoc Thai. 2006;89(11):184550.

20. Hui DS, Ko FW, Chan JK, To KW, Fok JP, Ngai JC, et al. Sleep-disordered breathing and continuous positive airway pressure compliance in a group of commercial bus drivers in Hong Kong. Respirology. 2006;11(6):723-30.

21. Liendo GR, Castro CL, Castro JR. Cansancio y somnolencia en conductores de omnibus 
interprovinciales: estudio comparativo entre formalidad e informalidad. Rev Peru Med Exp Salud Publica. 2010;27(2):187-94.

22. Pérez-Chada $D$, Videla AJ, O'Flaherty ME, Palermo $P$, Meoni J, Sarchi MI, et al. Sleep habits and accident risk among truck drivers: a cross-sectional study in Argentina. Sleep. 2005;28(9):1103-8.

23. Canani SF, John AB, Raymundi MG, Schönwald $S$, Menna Barreto SS. Prevalence of sleepiness in a group of Brazilian lorry drivers. Public Health. 2005;119(10):925-9.

24. Lemos LC, Marqueze EC, Sachi F, Lorenzi-Filho G, Moreno CRC. Síndrome da apneia obstrutiva do sono em motoristas de caminhão. J Bras Pneumol. 2009;35(6):500-6.

25. Santos EH, de Mello MT, Pradella-Hallinan M, Luchesi L, Pires ML, Tufik S. Sleep and sleepiness among Brazilian shift-working bus drivers. Chronobiol Int. 2004;21(6):881-8.

26. Moreno CR, Carvalho FA, Lorenzi C, Matuzaki LS, Prezotti S, Bighetti $P$, et al. High risk for obstructive sleep apnea in truck drivers estimated by the Berlin questionnaire: prevalence and associated factors. Chronobiol Int. 2004;21(6):871-9.

27. Macchi MM, Boulos Z, Ranney T, Simmons L, Campbell $S S$. Effects of an afternoon nap on nighttime alertness and performance in long-haul drivers. Accid Anal Prev. 2002;34(6):825-34.

28. Hartenbaum N, Collop N, Rosen IM, Phillips B, George CF, Rowley JA, et al. Sleep apnea and commercial motor vehicle operators: statement from the joint Task Force of the American College of Chest Physicians, American College of Occupational and Environmental Medicine, and the National Sleep Foundation. J Occup Environ Med. 2006;48(9 Suppl):S4-37.

29. Collop N, Hartenbaum N, Rosen I, Phillips B. Paying attention to at-risk commercial vehicle operators. Chest. 2006;130(3):637-9.

30. Durand G, Kales SN. Obstructive sleep apnea screening during commercial driver medical examinations: a survey of ACOEM members. J Occup Environ Med. 2009;51(10):1220-6.

31. Tippin J, Sparks J, Rizzo M. Visual vigilance in drivers with obstructive sleep apnea. J Psychosom Res. 2009;67(2):143-51.

32. Carmona BC, Capote GF, Botebol BG, García LP, Sánchez AA, Castillo GJ. Assessment of excessive day-time sleepiness in professional drivers with suspected obstructive sleep apnea syndrome. Arch Bronconeumol. 2000;36(8):436-40.

33. Philip P, Taillard J, Moore N, Delord S, Valtat C, Sagaspe $P$, et al. The effects of coffee and napping on nighttime highway driving: a randomized trial. Ann Intern Med. 2006;144(11):785-91.

34. Landström U, Akerstedt T, Byström M, Nordström B, Wibom R. Effect on truck drivers' alertness of a 30-min. exposure to bright light: a field study. Percept Mot Skills. 2004;98(3 Pt 1):770-6.

35. Jackson ML, Croft RJ, Owens K, Pierce RJ, Kennedy GA, Crewther D, et al. The effect of acute sleep deprivation on visual evoked potentials in professional drivers. Sleep. 2008;31(9):1261-9.

36. Leufkens TR, Lund JS, Vermeeren A. Highway driving performance and cognitive functioning the morning after bedtime and middle-of-the-night use of gaboxadol, zopiclone and zolpidem. J Sleep Res. 2009;18(4):38796.

37. Vakulin A, Baulk SD, Catcheside PG, Antic NA, van den Heuvel CJ, Dorrian J, et al. Effects of alcohol and sleep restriction on simulated driving performance in untreated patients with obstructive sleep apnea. Ann Intern Med. 2009;151(7):447-55.

38. Wilson RJ, Fang M, Cooper PJ, Beirness DJ. Sleepiness among night-time drivers: relationship to blood alcohol concentration and other factors. Traffic Inj Prev. 2006;7(1):15-22.

Artigo recebido em: 07/02/2011

Artigo aceito em: 05/04/2011 